\title{
Gender Affirming Voice Care: A Literature Review
}

Joseph Chang ${ }^{1}$, Katherine Yung ${ }^{2}$

\begin{abstract}
Aim and objective: Review recent advances and understanding of gender affirming voice care

Background:Treatment for gender dysphoria is increasingly recognized as medically necessary. Voice care is similarly as important as treatments focused on physical change.

Review results: This article reviews gender cues in communication as well as voice care options for voice masculinization and feminization. Voice masculinization techniques are poorly researched due to expectations that hormone replacement therapy (HRT) will result in adequate masculinization. HRT results are variable, however, and voice therapy and surgery are secondary options. Voice feminization has been better studied. While voice therapy and voice surgery are both options, voice therapy is often pursued first due to a low risk profile. Nonetheless, voice surgery techniques including Wendler glottoplasty (WG) and its variations are effective and safe.

Conclusion: Gender affirming voice care is critical in the treatment of gender dysphoria. HRT is often inadequate for voice masculinization. Further research in voice therapy and surgery options is needed. In voice feminization, voice therapy and voice surgery, particularly WG, have been effective in improving not only vocal pitch but also quality of life.

Clinical significance: Transgender individuals constitute a significant proportion of the population and frequently suffer from gender dysphoria. Gender affirming voice care is an essential component of treating gender dysphoria.

Keywords: Gender dysphoria, Hormone replacement therapy, HRT, Laryngoplasty, Trans voice, Vocal fold shortening and retrodisplacement of the anterior commissure (VFSRAC), Voice surgery, Voice therapy, Wendler.

International Journal of Head and Neck Surgery (2021): 10.5005/jp-journals-10001-1448
\end{abstract}

\section{BACKGROUND}

Transgender individuals experience a discordance between their own personal sense of gender and the sex assigned to them at birth. These individuals comprise a sizeable, $0.5-1.2 \%$ of the total population. ${ }^{1}$ Gender dysphoria is the term used to describe the distress from misalignment of self-identified and assigned gender. Treating gender dysphoria has been associated with decreased suicide attempt rates, ${ }^{2}$ improved quality of life, ${ }^{2,3}$ and increased rate of employment ${ }^{2}$ and pursuit of higher education. ${ }^{4}$ Given these health and socioeconomic benefits, insurance coverage for transgender services has been found to be cost effective, ${ }^{5}$ with an insignificant economic cost, ${ }^{6,7}$ and has been found to be medically necessary by numerous major medical associations including the American Medical Association (AMA), ${ }^{8}$ American Psychiatric Association (APA), ${ }^{9}$ American Academy of Family Physicians (AAFP) ${ }^{10}$ American Academy of Pediatrics (AACP), ${ }^{11}$ and American College of Obstetricians and Gynecologists (ACOG). ${ }^{12}$

While treatment for gender dysphoria has been available since the second half of the 20th century, ${ }_{1}^{13}$ recognition of the healthcare needs of this population have been increasing in more recent years. From 2012 to 2017, 12 states and the District of Columbia expanded their Medicaid coverage to include transition-related care. ${ }^{14}$ Additionally, the percent of Fortune 500 companies that have included transgender-inclusive health insurance has increased from $0 \%$ in 2009 to $71 \%$ in $2021 .{ }^{15}$

While many gender affirming treatments focus on physical appearance, voice and communication are similarly important to gender transition. In a national survey of 27,700 transgender individuals, 66 and $48 \%$ of transgender women respondents have had or want vaginoplasty or facial feminization surgery, respectively, compared to $62 \%$ of transgender women respondents who have had or want voice therapy. ${ }^{16}$
${ }^{1}$ Department of Head and Neck Surgery, The Permanente Medical Group, Santa Clara, California, USA

${ }^{2}$ San Francisco Voice \& Swallowing, San Francisco, California, USA

Corresponding Author: Department of Head and Neck Surgery, The Permanente Medical Group, Santa Clara, California, USA, Phone: 408-851-2950, e-mail: josephbchang@gmail.com

How to cite this article: Chang J, Yung K. Gender Affirming Voice Care: A Literature Review. Int J Head Neck Surg 2021;12(3):93-97.

Source of support: $\mathrm{Nil}$

Conflict of interest: None

This article reviews the recent advances and understanding of gender affirming voice care with a focus on voice surgery.

\section{Gender Cues in Communication}

High and low vocal pitch are commonly identified as indicators of masculinity and femininity. Normative ranges for pitch vary by source, with fundamental frequency of cisgender men and women generally in the range of $100-120$ and $190-220 \mathrm{~Hz}$, respectively. However, pitch is only one of many qualities known to affect gender identification and has been found to account for only $42 \%$ of variance in perception of speaker gender. ${ }^{17}$

Additional factors found to affect perception of gender according to research without high risk of bias include resonance, which describes the filtering effect of the upper aerodigestive tract on voice, articulation patterns, and intonation. ${ }^{17}$

Multiple gender communication cues must be addressed to effectively change gender perception. As an example, accurate gender identification in digitally modified voice samples was shown to fall to less than $34 \%$ when pitch and resonance were incongruent. ${ }^{18}$

(c) The Author(s). 2021 Open Access This article is distributed under the terms of the Creative Commons Attribution 4.0 International License (https://creativecommons. org/licenses/by-nc/4.0/), which permits unrestricted use, distribution, and non-commercial reproduction in any medium, provided you give appropriate credit to the original author(s) and the source, provide a link to the Creative Commons license, and indicate if changes were made. The Creative Commons Public Domain Dedication waiver (http://creativecommons.org/publicdomain/zero/1.0/) applies to the data made available in this article, unless otherwise stated. 


\section{Approach to Treatment}

Arguably more important than perceived vocal femininity or masculinity is the effect that treatment has on individuals' quality of life. Normative ranges and qualities exist for stereotypical male and female voices; however, these characteristics may not be congruent with every patient's own self-perception. Ultimately, quality of life has been shown to correlate with patients' perception of their own voice rather than the perception of listeners. ${ }^{19}$ As a result, treatment options may need to be adjusted for every patient to optimize outcomes.

In general, there are two treatment options for voice alteration, voice therapy and voice surgery. Voice therapy is able to address multiple communication cues and is noninvasive, whereas voice surgery primarily addresses pitch, is invasive, and therefore has associated small but significant medical risks. Consideration of risks and benefits in this situation often favors voice therapy as the first treatment for voice adjustment.

\section{Voice Masculinization}

Voice care for transgender men has been relatively less studied than care for transgender women due to the masculinizing effects of hormone replacement therapy (HRT) involving androgens. Multiple longitudinal studies have confirmed that fundamental frequency decreases within the first 12 months of starting HRT. ${ }^{20,21}$ However, the effect of HRT on voice is quite variable with ultimate decrease in pitch varying from 3 to 10 semitones in one prospective study and the onset of pitch decrease starting anywhere from 0 to 6 months after initiating $\mathrm{HRT}^{20}$

Unfortunately, voice change from HRT is not without risk. A meta-analysis of transgender men who underwent HRT found that $37 \%$ developed vocal instability and $27 \%$ lost vocal endurance. ${ }^{22} \mathrm{~A}$ retrospective study of 50 patients showed that $24 \%$ sought voice therapy while on HRT due to voice problems including vocal fatigue, instability, and strain with $10 \%$ seeking voice therapy for insufficient pitch lowering. ${ }^{21}$ Another meta-analysis confirmed that $16 \%$ of transgender patients undergoing testosterone therapy were not satisfied with their voice after hormone treatment. ${ }^{23}$

While voice therapy for voice masculinization is cited as a treatment option for patients with inadequate improvement after HRT, little data exist on the outcomes of voice therapy in the transgender male population. ${ }^{22}$ However, one study of 10 transgender men showed successful decrease in pitch after voice therapy with steady improvement in the self-perceived overall vocal masculinity up to 12 months after therapy. ${ }^{24}$

Surgery for pitch depression has been described using the type III thyroplasty, or relaxation laryngoplasty. In this surgery, pitch is decreased by shortening the vocal folds and reducing vocal fold tension. This can be performed by vertically incising both thyroid alae and depressing the central segment of thyroid cartilage containing the anterior vocal fold attachments to decrease the anteroposterior dimensions of the laryngeal framework. While type III thyroplasty has been described in mutational falsetto, ${ }^{25,26}$ only case reports of this surgery in transgender men have been published. ${ }^{27}$

\section{Voice Feminization}

Unlike hormone therapy for transgender men, hormone therapy for transgender women does not result in voice change. As a result, the treatments for voice feminization in the transgender population are more commonly pursued and better understood.

\section{Voice Therapy}

Voice therapy approaches vary widely with common targets including resonance, speaking pitch elevation, relaxation techniques, and intonation. ${ }^{28}$ Although this variation in approaches complicates standardized evaluation of voice therapy, a number of studies have shown that voice therapy is effective at improving listener perception of vocal femininity, ${ }^{29}$ self-rating of vocal femininity, ${ }^{30}$ satisfaction with voice, ${ }^{30}$ and quality of life. ${ }^{31}$

Yet, listener perception of vocal femininity ${ }^{29}$ and pitch elevation ${ }^{29,32}$ may not be maintained long term after voice therapy ends. A small study of five transwomen undergoing voice therapy showed that the initial average post-treatment pitch elevation of $59 \mathrm{~Hz}$ had decreased to $19 \mathrm{~Hz}$ by 15 months after therapy. ${ }^{29}$

\section{Voice Surgery}

Despite voice therapy, a number of individuals continue to have difficulty achieving or maintaining a desired pitch and choose to have additional surgical treatment. The percentage of individuals who choose to undergo surgery after voice therapy will certainly vary by center with those centers that offer voice surgery undoubtedly attracting a higher proportion of patients who will ultimately pursue surgery. Nonetheless, demand for surgery is considerable with $19 \%$ of patients in a national survey of transgender women showing interest in voice surgery, ${ }_{1}^{16}$ and an estimated $20 \%$ of transgender women who had completed voice therapy at one center going on to have voice surgery. ${ }^{33}$

Numerous surgeries have been developed for voice feminization. These surgeries include techniques to decrease vocal fold mass (laser reduction glottoplasty ${ }^{34,35}$ ), increase vocal fold tension [cricothyroid approximation (CTA)], and shorten vocal fold length [Wendler glottoplasty (WG) and feminization laryngoplasty $\left.{ }^{36,37}\right]$. Among these surgeries WG and CTA may be the best studied in the literature.

\section{Cricothyroid Approximation}

Cricothyroid approximation (CTA) is an open surgical technique in which the anteroinferior edge of the thyroid cartilage is brought into apposition with the anterior cricoid body with nonabsorbable sutures to recreate the vocal fold elongating effect of the cricothyroid muscle, thereby fixing the vocal folds in a permanently tensed state.

Multiple studies have confirmed successful elevation of pitch after CTA with reports ranging from 23 to $76 \mathrm{~Hz} .{ }^{38-40}$ Variation in reported pitch elevation may result from differences in evaluated voice samples, for example, sustained vowels versus standardized passage reading or spontaneous speech. Additional factors more specific to pitch variation after CTA include patient anatomy and time since surgical intervention. Cricothyroid joint configuration has been found to correlate with achievable pitch elevation with well-defined joints, type A joints, having greater pitch elevation than poorly defined or absent joints, type B and C joints. ${ }^{39}$

Additionally, two longitudinal studies of CTA have shown that pitch elevation effects are not durable on long-term follow-up. One study of 20 patients noted a $73 \mathrm{~Hz}$ pitch elevation at 6 months, which then dropped to $46 \mathrm{~Hz}$ at 24 months after surgery. ${ }^{40}$ Another study of 29 patients reported a $42 \mathrm{~Hz}$ pitch elevation at 6 months that fell to $23 \mathrm{~Hz}$ at 12 months with continued decreases in pitch elevation through the 4-year study period. ${ }^{38}$ 
Arguably more important than pitch elevation, quality of life, as measured by voice handicap index (VHI), has been found to improve after CTA. ${ }^{38}$ However, it is not clear that VHI adequately measures voice-related quality of life in the transgender population, potentially because anatomic abnormalities are not the cause of reduced quality of life in this population. In one study, despite being regularly misgendered on the phone, transgender participants had no disability on VHI. ${ }^{41}$ The trans woman voice questionnaire (TWVQ), previously known as the transsexual voice questionnaire (TVQ), has been developed and validated specifically to measure voice-related quality of life in the transgender woman population. ${ }^{42-44}$ To our knowledge, no studies have reported TWVQ results following CTA.

\section{Wendler Glottoplasty}

Wendler glottoplasty is a minimally invasive, endoscopic technique in which the medial aspects of the anterior vocal folds are denuded and sutured together (Fig. 1) to create an anterior glottic web (Fig. 2), thereby shortening the length of the vocal fold that is involved in vibration. Of note, there are slight variations to this technique including vocal fold shortening and retrodisplacement of the anterior commissure (VFSRAC), in which particular attention is paid to recreation of a tapered, funnel-shaped infraglottis to enhance smooth glottic air flow, ${ }^{45}$ as well another variation in which an additional step is taken to ablate the superior vocal fold surface, similar to laser reduction glottoplasty. ${ }^{38,46,47}$

Similar to CTA, numerous studies have reported successful pitch elevation following WG ranging from 20 to $81 \mathrm{~Hz} .{ }^{38,45,47,48}$ Variation in these outcomes may be due to variation in patient age and an additional pitch elevation effect from postoperative voice therapy. The studies from Kim and Mastronikolis et al. included postoperative voice therapy and reported the higher pitch elevation outcomes of 74 to $81 \mathrm{~Hz} .{ }^{45,47}$ Although no comparative statistics were performed between age groups, these two studies also reported greater pitch elevation in patients younger than 40 years. ${ }^{45,47}$ However, a study excluding the effects of voice therapy showed no correlation with age and postoperative pitch or change in pitch. ${ }^{48}$

Unlike CTA, pitch elevation after WG appears to be long lasting and improvement in quality of life specific to transgender women after WG is well documented. One study of patients undergoing WG showed similar pitch elevation from the 1 month postoperative evaluation to the last follow-up time point at 4 years. ${ }^{38}$ Additionally, multiple studies have found improvement in $\mathrm{TWVQ}^{46,48}$ as well as $\mathrm{VHI}{ }^{38,45,48}$
In the current paradigm in which voice therapy is often pursued prior to voice surgery, some may consider voice surgery as an option only for those who "fail" voice therapy. On the contrary, a recent study of voice therapy compared to combined voice therapy followed by WG suggests that addition of WG further elevates pitch and improves quality of life even in those patients who objectively succeed after voice therapy. After voice therapy, patients who chose to pursue additional WG had no statistically significant difference in average speaking fundamental frequency or degree of pitch elevation compared to those who chose not to pursue additional WG, with 20 and $15 \mathrm{~Hz}$ elevations in pitch, respectively. Nonetheless, pitch elevation and improvement in TWVQ were nearly three times higher after combined treatment compared to voice therapy only. ${ }^{31}$

\section{Comparison of Techniques}

Due to lack of standardization in voice measurements and timing of evaluations, comparisons between CTA and WG are best made by studies specifically designed to compare these two techniques with uniform methods. Two such studies exist. Mora et al. found that self-perceived femininity and pitch elevation were statistically higher in individuals who underwent WG compared to those who underwent CTA. ${ }^{38}$ Kelly et al. similarly reported statistically significant improvement in satisfaction with voice in individuals who underwent WG, but not those individuals who underwent CTA. Moreover, there was a significantly lower rate of self-reported dysphonia in patients who underwent WG compared to CTA. These findings in combination with the greater ability to revise WG than CTA led these authors to stop performing CTA at their institution in favor of WG. ${ }^{33}$

\section{Surgical Complications}

Historically, there have been concerns regarding the viability of voice surgery as a treatment for voice feminization due to reports of postsurgical dysphonia, dysphagia, and infection. ${ }^{49}$ Indeed, CTA has been found to have a $7 \%$ rate of wound infection ${ }^{38}$ in one series and $29 \%$ rate of postoperative dysphagia in another series. ${ }^{40}$ On the other hand, those same series reported no change in voice quality on perceptual evaluation using the GRBAS (Grade, Roughness, Breathiness, Asthenia, Strain) score ${ }^{38}$ and a minimal change in average jitter from 0.6 to $0.7 \%{ }^{40}$

Wendler glottoplasty has a seemingly even lower rate of postoperative complications. While web dehiscence has been reported at rates of $5-13 \%,{ }^{31,38}$ no reports of postoperative
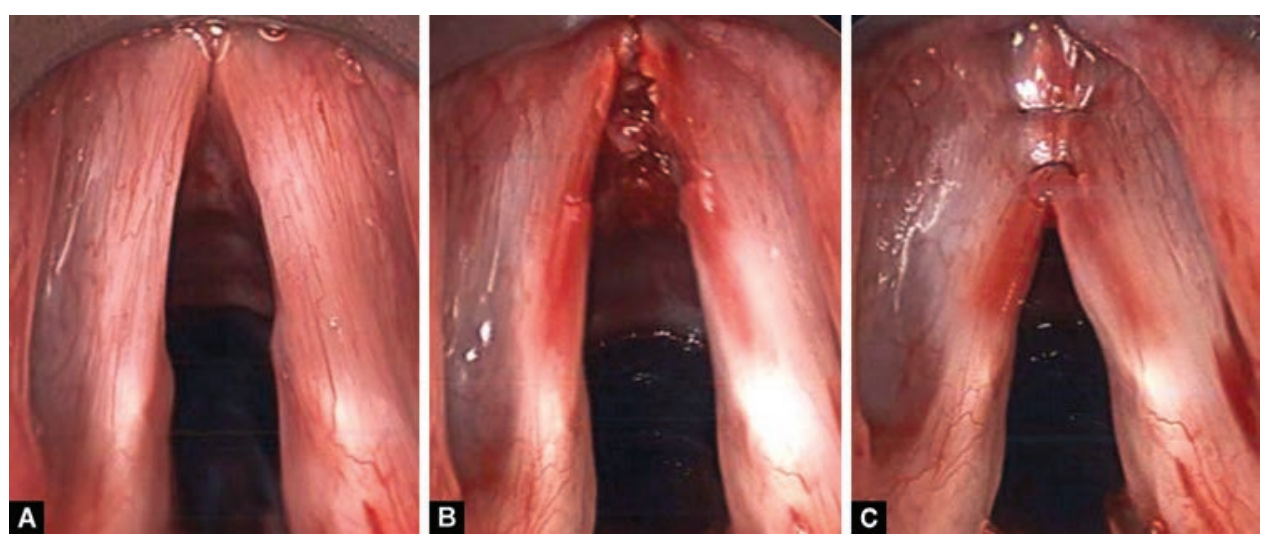

Figs $1 \mathrm{~A}$ to C: Wendler glottoplasty technique. Prior to incision (A), after removal of the anterior medial vocal fold mucosa (B), and after suturing the anterior vocal folds together at the completion of surgery (C). 


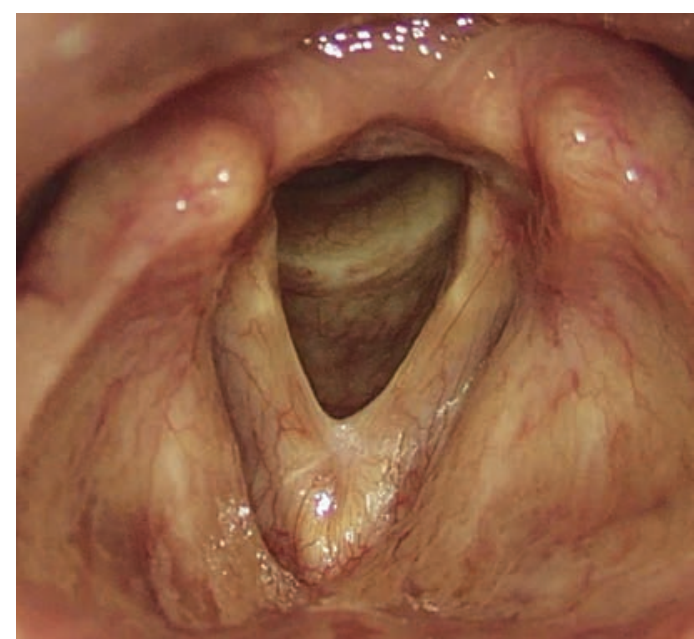

Fig. 2: Wendler glottoplasty postoperative appearance. Well-formed anterior glottic web at 3 months after surgery.

infection or dysphagia have been identified on systematic review of the WG literature. ${ }^{50}$ Some data exist to suggest that glottoplasty causes postoperative dysphonia; a meta-analysis of three studies including 70 patients reported that auditory perceptual evaluation of overall voice quality graded on the GRBAS scale worsened by 0.44 after glottoplasty. ${ }^{50}$ This degree of worsening represents a half-step worsening on a four-point scale with gradations of normal, mild, moderate, and severe dysphonia; the clinical significance of this degree of change is unclear. Moreover, larger studies of objective acoustic measures show no worsening of dysphonia. In a series of 28 patients, no change in cepstral peak prominence, cepstral index of dysphonia, noise to harmonic ratio, or soft phonation index was detected after glottoplasty. ${ }^{48}$ Similarly, another study of 362 patients undergoing glottoplasty showed no change in noise to harmonic ratio and slight improvement in soft phonation index. ${ }^{45}$

Although arguments could be made regarding the sensitivity of perceptual evaluation versus acoustic measurements and the clinical significance of a 0.44 point worsening in dysphonia, it is possible that the difference in postoperative dysphonia may be related to differences in technique. The three studies utilized in the meta-analysis by Aires et al. that report worsening in perceptual measures of voice all report concomitant ablation of the superior vocal fold surface in addition to creation of an anterior glottic web, $^{38,46,47}$ in essence a combination of laser reduction glottoplasty and WG. In contrast, the two previously cited studies showing no change in acoustic measures only created an anterior glottic web and did not ablate additional vocal fold tissue. ${ }^{45,48}$

While the risks and benefits of voice surgery compared to voice therapy can be debated, recent data shows that WG can be routinely performed with minimal morbidity with the acknowledgement that any surgery when performed poorly can result in serious complications.

\section{Conclusion}

Treatment for gender dysphoria is increasingly recognized as medically necessary. In addition to treatments focused on physical change, treatments for changing voice and communication are also essential. In the transgender male population, HRT is helpful in masculinizing the voice although its effects are variable. Unfortunately, voice therapy and voice surgery for voice masculinization is poorly understood and in need of further research. In the transgender female population, the efficacy of voice therapy and voice surgery is well documented in the literature, although approaches and techniques vary. Wendler glottoplasty has been shown to be a safe and durable intervention for voice feminization with minimal to no expected postoperative dysphonia.

\section{References}

1. Winter S, Diamond M, Green J, et al. Transgender people: health at the margins of society. Lancet 2016;388((10042)):390-400. DOI: 10.1016/s0140-6736(16)00683-8

2. Murad MH, Elamin MB, Garcia MZ, et al. Hormonal therapy and sex reassignment: a systematic review and meta-analysis of quality of life and psychosocial outcomes. Clin Endocrinol (Oxf) 2010;72(2):214-231. DOI: 10.1111/j.1365-2265.2009.03625.x.

3. Ainsworth TA, Spiegel JH. Quality of life of individuals with and without facial feminization surgery or gender reassignment surgery. Qual Life Res 2010;19(7):1019-1024. DOI: 10.1007/s11136-010-9668-7

4. Rakic Z, Starcevic V, Maric J, et al. The outcome of sex reassignment surgery in Belgrade: 32 patients of both sexes. Arch Sex Behav 1996;25(5):515-525. DOI: 10.1007/BF02437545

5. Padula WV, Heru S, Campbell JD. Societal implications of health insurance coverage for medically necessary services in the US transgender population: a cost-effectiveness analysis. J Gen Intern Med 31(4):394-401. DOI: 10.1007/s11606-015-3529-6

6. State of California, Department of Insurance. Economic Impact Assessment, Gender Nondiscrimination in Health Insurance. April 13, 2012. REG-2011-00023.

7. Human Rights Campaign Foundation. San Francisco Transgender Benefit. Available at: https://www.thehrcfoundation.org/ professional-resources/san-francisco-transgender-benefit. [Accessed 11 December 2021].

8. American Medical Association. Issue brief: health insurance coverage for gender-affirming care of transgender patients. Available at: https://ama-assn.org/system/files/2019-03/transgender-coverageissue-brief.pdf.

9. American Psychiatric Association. APA official actions: position statement on discrimination against transgender and gender diverse individuals. Available at: https://www.psychiatry.org/File\%20Library/ About-APA/Organization-Documents-Policies/Policies/Position2018-Discrimination-Against-Transgender-and-Gender-DiverseIndividuals.pdf

10. Ellis L, Brammer W, Echols B, et al. Resolution No. 1004. Available at: https://aafp.org/dam/AAFP/documents/about_us/special_ constituencies/2012RCAR_Advocacy.pdf.

11. Rafferty J. Ensuring comprehensive care and support for transgender and gender-diverse children and adolescents. Pediatrics 2018;142(4):e20182162. DOI: 10.1542/peds.2018-2162

12. Health care for transgender and gender diverse individuals: ACOG committee opinion, number 823. Obstet Gynecol 2021;137(3): e75-e88. DOI: 10.1097/AOG.0000000000004294

13. Coleman E, Bockting W, Botzer M, et al. Standards of care for the health of transsexual, transgender, and gender-nonconforming people, version 7. Int J Transgenderism 2012;13(4):165-232. DOI: 10.1080/15532739.2011.700873

14. Baker KE. The future of transgender coverage. $\mathrm{N}$ Engl J Med 2017;376(19):1801-1804. DOI: 10.1056/NEJMp1702427

15. Human Rights Campaign Foundation. Corporate equality index 2021. Available at: https://reports.hrc.org/corporate-equalityindex-2021.

16. James SE, Herman JL, Rankin S, et al. The report of the 2015 U.S. transgender survey. 2016. Available at: https://transequality.org/ sites/default/files/docs/usts/USTS-Full-Report-Dec17.pdf.

17. Leung Y, Oates J, Chan SP. Voice, articulation, and prosody contribute to listener perceptions of speaker gender: a systematic review and meta-analysis. J Speech Lang Hear Res 2018;61(2):266-297. DOI: 10.1044/2017_JSLHR-S-17-0067 
18. Hillenbrand JM, Clark MJ. The role of $f(0)$ and formant frequencies in distinguishing the voice of men and women. Atten Percept Psychophys 2009;71(5):1150-1166. DOI: 10.3758/APP.71.5.1150

19. Hancock AB, Krissinger J, Owen K. Voice perceptions and quality of life of transgender people. J Voice 2011;25(5):553-558. DOI: 10.1016/j. jvoice.2010.07.013

20. Hancock AB, Childs KD, Irwig MS. Trans male voice in the first year of testosterone therapy: make no assumptions. J Speech Lang Hear Res 2017;60(9):2472-2482. DOI: 10.1044/2017_jslhr-s-16-0320

21. Nygren U, Nordenskjold A, Arver S, et al. Effects on voice fundamental frequency and satisfaction with voice in trans med during testosterone treat-a longitudinal study. J Voice 2016;30(6):766.e23-e34. DOI: 10.1016/j.jvoice.2015.10.016

22. Azul D, Nygren U, Sodersten M, et al. Transmasculine people's voice function: a review of the currently available evidence. J Voice 2017;31(2):261.e9-23. DOI: 10.1016/j.jvoice.2016.05.005

23. Ziegler A, Henke T, Wiedrick J, et al. Effectiveness of testosterone therapy for masculinizing voice in transgender patients: a meta-analytic review. Int J Transgenderism 2018;19(1):25-45. DOI: 10.1080/15532739.2017.1411857

24. Mills M, Stoneham G, Davies S. Toward a protocol for transmasculine voice: a service evaluation of the voice and communication therapy group program, including long-term follow-up for trans men at the London gender identity clinic. Transgend Health 2019;4(1):143-151. DOI: 10.1089/trgh.2019.0011

25. Li GD, Mu L, Yang S. Acoustic evaluation of Isshiki type III thyroplasty for treatment of mutational voice disorders. J Laryngol Otol 1999;113(1):31-34. DOI: 10.1017/s0022215100143099

26. Remacle M, Matar N, Verduyckt I, et al. Relaxation thyroplasty for mutational falsetto treatment. Ann Otol Rhinol Laryngol 2010;119(2):105-109. DOI: 10.1177/000348941011900207

27. Saito $Y$, Nakamura K, Itani S, et al. Type 3 thyroplasty for a patient with female-to-male gender identity disorder. Case Rep Otolaryngol 2018;2018:4280381. DOI: 10.1155/2018/4280381

28. Hancock AB, Garabedian LM. Transgender voice and communication treatment: a retrospective chart review of 25 cases. Int J Commun Disord 2013;48(1):54-65. DOI: 10.1111/j.1460-6984.2012.00185.x

29. Gelfer MP, Tice RM. Perceptual and acoustic outcomes of voice therapy for mail-to-female transgender individuals immediately after therapy and 15 months later. J Voice 2013;27(3):335-347. DOI: 10.1016/j.jvoice.2012.07.009

30. Carew L, Dacakis G, Oates J. The effectiveness of oral resonance therapy on the perception of femininity of voice in male-tofemale transsexuals. J Voice 2007;21(5):591-603. DOI: 10.1016/j. jvoice.2006.05.005

31. Brown SK, Chang J, Hu S, Sivakumar G, et al. Addition of Wendler glottoplasty to voice therapy improves trans female voice outcomes. Laryngoscope 2020;131(7):1588-1593. DOI: 10.1002/lary.29050

32. Dacakis G. Long-term maintenance of fundamental frequency increases in male-to-female transsexuals. J Voice 2000;14(4):549-556. DOI: 10.1016/s0892-1997(00)80010-7

33. Kelly V, Hertegård S, Eriksson J, et al. Effects of gender-confirming pitch-raising surgery in transgender women a long-term follow-up study of acoustic and patient-reported data. JVoice 2019;33(5):781-791. DOI: 10.1016/j.jvoice.2018.03.005

34. Kocak I, Akpinar ME, Cakir ZA, et al. Laser reduction glottoplasty for managing androphonia after failed cricothyroid approximation surgery. J Voice 2010;24(6):758-764. DOI: 10.1016/j.jvoice.2009.06.004
35. Orloff LA, Mann AP, Damrose JF, et al. Laser-assisted voice adjustment (LAVA) in transsexuals. Laryngoscope 2006;116(4):655-660. DOI: 10.1097/01.mlg.0000205198.65797.59

36. Kunachak S, Prakunhungsit S, Sujjalak K. Thyroid cartilage and vocal fold reduction: a new phonosurgical method for male-to-female transsexuals. Ann Otol Rhinol Laryngol 2000;109(11): 1082-1086. DOI: 10.1177/000348940010901116

37. Thomas JP, Macmillan C. Feminization laryngoplasty: assessment of surgical pitch elevation. Eur Arch Otorhinolaryngol 2013;270(10): 2695-2700. DOI: 10.1007/s00405-013-2511-3

38. Mora E, Cobeta I, Becerra A, et al. Comparison of cricothyroid approximation and glottoplasty for surgical voice feminization in male-to-female transsexuals. Laryngoscope 2018;128(9):2101-2109. DOI: 10.1002/lary. 27172

39. Tschan S, Honegger F, Storck C. Cricothyroid joint anatomy as a predicting factor for success of cricoid-thyroid approximation in transwomen. Laryngoscope 2016;126(6):1280-1283. DOI: 10.1002/lary. 25518

40. Yang CY, Palmer AD, Murray KD, et al. Cricothyroid approximation to elevate vocal pitch in male-to-female transsexuals: results of surgery. Ann Otol Rhinol Laryngol 2002;111(6):477-485. DOI: 10.1177/000348940211100602

41. T'Sjoen G, Moerman M, Van Borsel J, et al. Impact of voice in transsexuals. Int J Transgenderism 2006;9(1):1-7. DOI:10.1300/ J485v09n01_01

42. Dacakis G, Davies S, Oates JM, et al. Development and preliminary evaluation of the transsexual voice questionnaire for male-to-female transsexuals. J Voice 2013;27(3):312-20. DOI: 10.1016/j. jvoice.2012.11.005

43. Dacakis G, Oates JM, Douglas JM. Exploring the validity of the transsexual voice questionnaire (male-to-female): do TVQ ${ }^{\mathrm{MtF}}$ scores differentiate between MtF women who have had gender reassignment surgery and those who have not? Int J Transgenderism 2016;17(3-4):124-130. DOI:10.1080/15532739.2016.1222922

44. Davies SM, Johnston JR. Exploring the validity of the transsexual voice questionnaire for male-to-female transsexuals. Can J Speech-Lang Pathol Audiol 2015;39(1):40-51.

45. Kim HT. A new conceptual approach for voice feminization: 12 years of experience. Laryngoscope 2017;127(5):1102-1108. DOI: 10.1002/lary.26127

46. Casado JC, O'Connor C, Angulo MS, et al. Wendler glottoplasty and voice-therapy in male-to-female transsexuals: results in pre and post-surgery assessment. Acta Otorrinolaringol Esp 2016;67(2):83-92. DOI: 10.1016/j.otorri.2015.02.003

47. Mastronikolis NS, Remacle M, Biagini M, et al. Wendler glottoplasty: an effective pitch raising surgery in male-to-female transsexuals. J Voice 2013;27(4):516-522. DOI: 10.1016/j.jvoice.2013.04.004

48. Chang J, Brown SK, Hu S, et al. Effect of Wendler glottoplasty on acoustic measures of voice. Laryngoscope 2021;131(3):583-586. DOI: 10.1002/lary.28764

49. Davies S, Papp VG, Antoni C. Voice and communication change for gender nonconforming individuals: giving voice to the person inside. Int J Transgenderism 2015;16(3):117-159. DOI: 10.1080/15532739.2015.1075931

50. Aires MM, Marinho CB, Souza CSC. Effect of endoscopic glottoplasty on acoustic measures and quality of voice: a systematic review and meta-analysis. J Voice 2020;S0892-1997(20)30420-3. DOI: 10.1016/j. jvoice.2020.11.005 